\title{
Counting the Number of Disconnected Labeled Graphs of Order Five without Paralel Edges
}

\author{
Wamiliana ${ }^{1, *}$, Amanto $^{1}$, Grita Tumpi Nagari ${ }^{1}$
}

\begin{abstract}
Given a graph $\mathrm{G}(\mathrm{V}, \mathrm{E})$ with $\mathrm{n}$ vertices and $\mathrm{m}$ edges, where every vertex is labeled, there are a lot of possible graphs that can be constructed, either connected graphs or disconnected, simple or not simple. A graph $\mathrm{G}(\mathrm{V}, \mathrm{E})$ is called as a connected graph if there exists at least one path between every pair of vertices in $G$, and otherwise, $G$ is disconnected. A graph $G$ is called as a labeled graph if every node/vertex and or every edge is labeled. In this research, we are concerning about a graph where every vertex is labeled. Parallel edges are two edges or more which have the same end points. In this research we found that the number of disconnected labeled graph without parallel edges for $n=5$ and $m \geq 1$ can be obtained with the following formula:

$N\left(G^{n}{ }^{\mathrm{a}, \mathrm{m}}\right)=\left(\begin{array}{c}m+4 \\ 4\end{array}\right)+\left\{10 \times\left(\begin{array}{c}m+d \\ 4\end{array}\right)\right\}+\left[45 \times\left(\begin{array}{c}m+2 \\ 4\end{array}\right)\right\}+\{120 \times$ $\left.\left(\begin{array}{c}m+1 \\ 4\end{array}\right)\right\}+\left\{85 \times\left(\begin{array}{c}m \\ 4\end{array}\right)\right\}+\left\{30 \times\left(\begin{array}{c}m-1 \\ 4\end{array}\right)\right\}+\left\{5 \times\left(\begin{array}{c}m-2 \\ 4\end{array}\right)\right\} . \quad \mathrm{N}\left(G^{\mathrm{m}, \mathrm{m}}\right)$ is the number of disconnected labeled graph without parallel edges for $n=5$ and $m \geq 1$
\end{abstract}

Keywords - counting graph, labeled graph, disconnected, parallel edges

\section{INTRODUCTION}

A fter Euler gave the solution for the Konigsberg's Bridge problem in 1736, Graph Theory had emerged as one important tool for solving daily life problems. In 1992 [1] provided a comprehensive account of applications of graph theory in the area of engineering (electrical, civil, and industrial), operations research and science. In science, for instance, he mentioned that in the modeling of phylogeny construction, the spanning tree concept was employed. Phylogeny is the tree that represents the relationship between the certain species living today with its ancestors. Related with the connection networks, in 2009 [2] explored and exposed the use of graph theoretic concepts in interconnection networks.

Given $\mathrm{n}$ vertices and $\mathrm{m}$ edges, where every vertex is labeled, there are a lot of possible graphs that can be constructed, either connected or disconnected, simple or not simple. Some researcher had already done some works related with the graph counting; for example in 1874 [3] presented that the tree structure can also be used to represent the hydrocarbon structure $\mathrm{C}_{n} \mathrm{H}_{2 n+2}$ and the problem of counting the number of rooted trees with $n$ vertices is equivalent with the problem of finding the number of isomers, and in 1964, [4] employed graph theory in order to obtain some chemical structures which are isomorphic to certain graph structures.

In this paper we will discuss the formula for counting the number of disconnected labeled graphs for graph with order five.

${ }^{1}$ Department of Mathematics, Faculty of Mathematics and Natura Sciences, Universitas Lampung, Jalan Prof. Soemantri Brojonegoro No. 1, Bandar Lampung, Indonesia.

${ }^{*}$ Correspondence Author, email: wamiliana.1963@fmipa.unila.ac.id

Tel.:+62-721-704625; fax.:+62-721-704625.

\section{BRIEF REVIEW ON GRAPH ENUMERATION}

As mention before, Cayley in 1874 investigated the numbers of hydrocarbon structure and this problem related to problem of counting the number of hydrocarbon structure, in 1973 Harary and Palmer gave the method for counting and labeling graph [5], and the method are use widely until now. In 1997 and 1999 Stanley provided generating function to obtain expressions or formulas in combinatorics [6,7], and in 1994 Wilf provided an introduction to various combinatorial counting techniques [8]. Agnarsson and Greenlaw in 2007 investigated the counting of simple graphs and derived the formula for counting the number of graphs. The formula consists of four parts, the first and the second are for counting the number of graphs without loops, and the third and fourth parts are for counting the number of graphs if the number of edges is given [9]. However, there is no formula for finding a graph with a special property such as connectivity, planarity, and others. Therefore, in this paper we will discuss about the formula for counting labeled disconnected graphs with order five without paralel edges.

\section{OBSERVATION AND INVESTIGATION}

Assume that $m$ is the number of edges, $g$ is the number of loopless edges, and $\ell_{[}$is the number of $i$-loop incidence to vertex $p$, with $i=0,1,2, \ldots$, and $p=1,2,3, \ldots, \mathrm{n}$.

Then $\left.m=g+\sum_{l=\downarrow}^{m-g}\right] \cdot \ell_{l}, \jmath \in \mathbb{N} \cup\{0\}$.

For example, at Fig. 1 we get, $g=1, \ell_{1}=2, \ell_{2}=1$

Then, $m=g+2 \ell_{1}+\ell_{2}=1+2+2=5$.

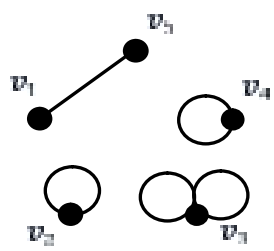

Fig. 1 Example of graph with $g=1, \ell_{1}=2, \ell_{2}=1$

In order to enumerate the number of disconnected labeled graphs without parallel edges (loops are allowable) we do the observation by finding/constructing the patterns and then calculate the numbers of different graphs in every pattern. Since there are many patterns formed, we only draw some examples of the patterns. The number of graphs 
is given in the tables. We want to mention here that we do observe and and investigate the graph for the number of edges given $\mathrm{m}$ is more than 10 , but since the graph continuing the patterns, then we stop observing and investigating for $\mathrm{m}$ more than 10 (since if we add more edges and paralel edges are not allowed to be constructed, then the patterns of the graph is like the patterrn already constructed, only the number of loops increases).

\section{A. Disconnected Labeled Graph Without Parallel Edges} for $n=5, m \geq 1,1 \leq g \leq 6$, and $\ell_{l}=0$ with $i=1,2, \ldots$

Among of the patterns observed, here we draw three of the patterns for disconnected labeled graph without parallel edges for $n=5, m \geq 1,1 \leq g \leq 6$, and $\ell_{l}=0$ with $i=$ $1,2, \ldots$

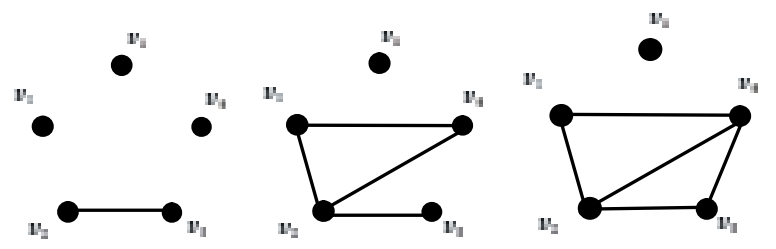

Fig. 2 Three of the patterns for $n=5,1 \leq m \leq 6,1 \leq g \leq 6$, and $\ell_{\mathrm{i}}=0$

The graphs obtained are graphs without loops, and the following table shows the result of numbers of graphs obtained. Note that in these patterns, the maximum number of edges only 6 .

Table 1. The number of disconnected labeled graph without parallel edges for $n=5, m \geq 1,1 \leq g \leq 6$, and $\ell_{i}=0$ with $i=1,2, \ldots$

$\begin{array}{lllllll}\text { The number of graph } & 10 & 45 & 120 & 85 & 30 & 5\end{array}$

B. Disconnected Labelled Graph Without Parallel Edges for $n=5, m \geq 1, g=0$, and $1 \leq \ell_{l} \leq$ with $i=1,2, \ldots$,

Among of the patterns observed, here we draw two of the patterns for disconnected labeled graph without parallel edges $n=5, m \geq 1, g=0$, and $1 \leq \ell_{l} \leq 5$ with $i=1,2, \ldots$

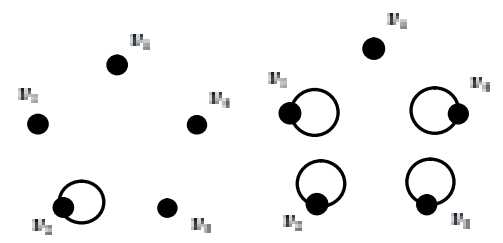

Fig. 3. Two of the patterns for $n=5, m \geq 1, g=0$, and $1 \leq \ell_{\mathrm{i}} \leq$

The graphs obtained by those patterns are graphs with loops only (all edges constitute loops), and the following table shows the result of numbers of graphs obtained

Table 2. The number of disconnected labeled graph without parallel edges for $n=5, m \geq 1, g=0$, and $1 \leq \ell \leq 5$ with $[=1,2$,

\begin{tabular}{|c|c|c|c|c|c|c|c|c|c|c|}
\hline$m$ & 1 & 2 & 3 & 4 & 5 & 6 & 7 & 8 & 9 & 10 \\
\hline $\begin{array}{c}\text { The } \\
\text { number } \\
\text { of graph }\end{array}$ & 5 & 15 & 35 & 70 & 126 & 210 & 330 & 495 & 715 & 1011 \\
\hline
\end{tabular}

C. Disconnected Labeled Graph Without Parallel Edges for $n=5, m \geq 1,1 \leq g \leq 6$, and $0 \leq \ell_{l} \leq 5$ with $i=1,2, \ldots$

Among of the patterns observed, here we draw two of the patterns for disconnected labeled graph without parallel edges $n=5, m \geq 1,1 \leq g \leq 6$, and $0 \leq \ell_{l} \leq 5$ with $i=1,2, \ldots$
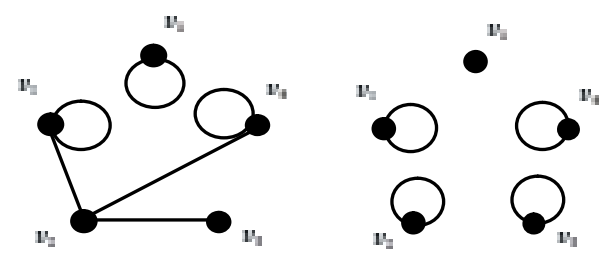

Figure 4. Two of the patterns for $n=5, m \geq 1,1 \leq g \leq 6$, and $0 \leq \ell_{i} \leq 5$

The graphs obtained by those patterns are graphs that contain loops or/and edges, and the following table shows the result of numbers of graphs obtained

Table 3. The number of disconnected labeled graph without parallel edges for $n=5, m \geq 1,1 \leq g \leq 6$, and $0 \leq \ell_{i} \leq 5, i=1,2, \ldots$

\begin{tabular}{llllllll}
\hline \hline $\mathbf{g}$ & $\mathbf{1}$ & $\mathbf{2}$ & $\mathbf{3}$ & $\mathbf{4}$ & $\mathbf{5}$ & $\mathbf{6}$ & $\begin{array}{l}\text { Total } \\
\text { graphs }\end{array}$ \\
\hline 2 & 50 & - & - & - & - & - & 50 \\
3 & 150 & 225 & - & - & - & - & 375 \\
4 & 350 & 675 & 600 & - & - & - & 1625 \\
5 & 700 & 1575 & 1800 & 425 & - & - & 4500 \\
6 & 1260 & 3150 & 4200 & 1275 & 150 & - & 10035 \\
7 & 2100 & 5670 & 8400 & 2975 & 450 & 25 & 19620 \\
8 & 3300 & 9450 & 15120 & 5950 & 1050 & 75 & 34945 \\
9 & 4950 & 14850 & 25200 & 10710 & 2100 & 175 & 57985 \\
10 & 7150 & 22275 & 39600 & 17850 & 3780 & 350 & 91005 \\
\hline \hline
\end{tabular}

Table 1 is table of disconnected graphs of order five without paralel edges and there are no loops; Table 2 is the table of disconnected graphs of order five without paralel edges and all the edges constitute loops. Table 3 is the table of disconnected graphs of order five without paralel edges where some edges are loops and some are not. By merging Table 1 and Table 3, we get the following table :

Tabel 4. The number of disconnected labeled graph without parallel edges for $n=5, m \geq 1,1 \leq g \leq 6$, dan $0 \leq \ell_{\mathrm{i}} \leq 5,[=0,1,2, \ldots, 9$

\begin{tabular}{llllllll}
\hline \begin{tabular}{l}
\hline $\mathbf{g}$ \\
$\mathbf{m}$
\end{tabular} & $\mathbf{1}$ & $\mathbf{2}$ & $\mathbf{3}$ & $\mathbf{4}$ & $\mathbf{5}$ & $\mathbf{6}$ & $\begin{array}{l}\text { Total } \\
\text { graphs }\end{array}$ \\
\hline 1 & 10 & - & - & - & - & - & 10 \\
2 & 50 & 45 & - & - & - & - & 95 \\
3 & 150 & 225 & 120 & - & - & - & 495 \\
4 & 350 & 675 & 600 & 85 & - & - & 1710 \\
5 & 700 & 1575 & 1800 & 425 & 30 & - & 4530 \\
6 & 1260 & 3150 & 4200 & 1275 & 150 & 5 & 10040 \\
7 & 2100 & 5670 & 8400 & 2975 & 450 & 25 & 19620 \\
8 & 3300 & 9450 & 15120 & 5950 & 1050 & 75 & 34945 \\
9 & 4950 & 14850 & 25200 & 10710 & 2100 & 175 & 57985 \\
10 & 7150 & 22275 & 39600 & 17850 & 3780 & 350 & 91005 \\
\hline \hline
\end{tabular}




\section{THE FORMULA OF THE PATERNS}

Denote $\mathrm{N}\left(G_{n, m, y}^{\prime}\right)$ as the number of disconnected labeled graphs without paralel edges with $n$ vertices, m edges, and $g$, where $g$ is the number of non loop edges that makes the graph disconnected. If $g$ is larger than 6 than the graph is either has paralel edges or becomes a connected graph. Therefore, the maximum number for $\mathrm{g}$ is 6 .

Theorem 1: $\mathrm{N}\left(G_{\square, m, \mathrm{~L}}^{\prime}\right)=\left(\begin{array}{c}\mathrm{m}+4 \\ 4\end{array}\right) ; m \geq 1$

Proof :

From Table 2 we get the following sequence of numbers :

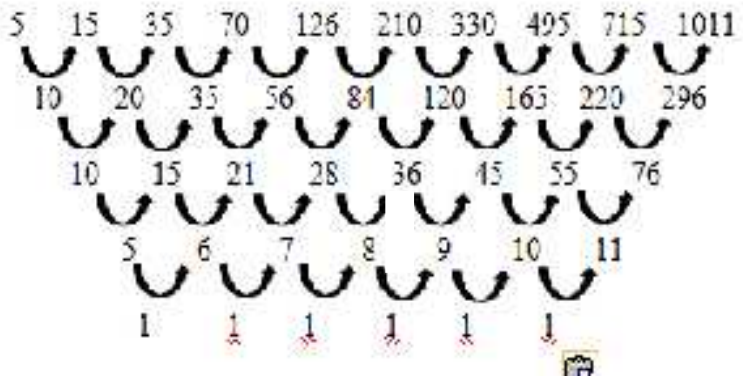

Since the fixed difference in the sequence occurs on the fourth order, the sequence is the arithmetics sequence of order four. Thus, we can construct an equation of order four as follow: $u_{m}=A m^{4}+B m^{3}+C m^{2}+D+E$

By substituting $\mathrm{m}=1,2,3,4$, and 5 to equation (2) we get the following :

$$
\begin{aligned}
& m=1 \rightarrow 5=A+B+C+D+E \\
& m=2 \rightarrow 15=16 A+8 B+4 C+2 D+E \\
& m=3 \rightarrow 35=81 A+27 B+9 C+3 D+E \\
& m=4 \rightarrow 70=256 A+64 B+16 C+4 D+E \\
& m=5 \rightarrow 126=625 A+125 B+25 C+5 D+E
\end{aligned}
$$

By applying elimination and substitution processes to the above equations, we ge the following :

$$
\begin{aligned}
u_{m}= & \frac{1}{24} m^{4}+\frac{10}{24} m^{2}+\frac{35}{24} m^{2}+\frac{50}{24} m+1 \\
= & \frac{1}{2}\left(m^{4}+m^{4}+m^{2}+m+24\right) \\
= & \frac{1}{2}(m+4)(m+3)(m+2)(m+1) \\
= & \frac{(m+4)(m+3)(m+2)(m+1)(m !)}{(4 \times 3 \times 2 \times 1)(m !)} \\
= & \left(\begin{array}{c}
m+4 \\
4
\end{array}\right)
\end{aligned}
$$

Therefore $\mathrm{N}\left(G_{5, \mathrm{~m}, 0}^{\prime}\right)=\left(\begin{array}{c}m+q \\ q\end{array}\right) ; m \geq 1$

Theorems 2 to 7 obtained when the number of $g=1,2,3,4,5$, 6 as follow :

Theorem 2: For $\mathrm{g}=1, \mathrm{~N}\left(G_{\mathrm{D}, \mathrm{m}, 1}^{\prime}\right)=10 \times\left(\begin{array}{c}\mathrm{m+1} \\ 4\end{array}\right), m \geq 1$

Proof :

From the first column of Table 4, we get the following sequence of numbers :

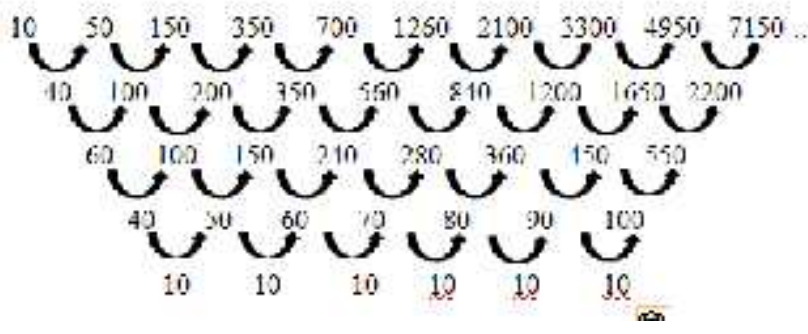

Since the fixed difference in the sequence occurs on the fourth order, the sequence is the arithmetics sequence of order four, and by similar approach of the proof of Theorem 1 we get the formula.

Theorem 3 : For $\mathrm{g}=2, \mathrm{~N}\left(G_{5, m, 2}^{\prime}\right)=45 \times\left(\begin{array}{c}m+2 \\ 4\end{array}\right), m \geq 2$ Proof :

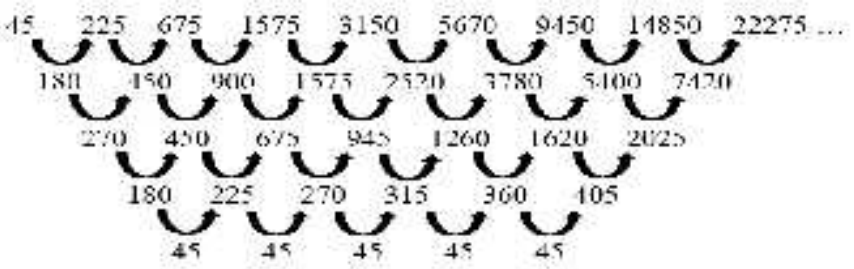

The proof is similar with the previous theorems, and since the fixed difference in the sequence occurs on the fourth order, the sequence is the arithmetics sequence of order four, and by similar approach we get the formula.

Theorem 4 : For $\mathrm{g}=3, \mathrm{~N}\left(G^{\mathrm{I}, \mathrm{m}, \mathrm{I}}\right)=120 \times\left(\begin{array}{c}m+1 \\ 4\end{array}\right), m \geq 3$ Proof :

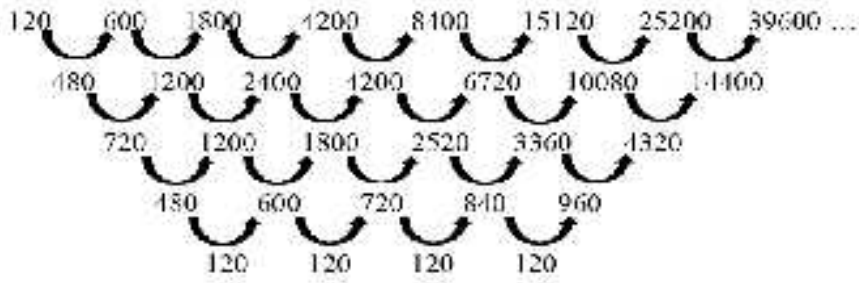

Again, since the fixed difference in the sequence occurs on the fourth order, the sequence is the arithmetics sequence of order four, and by similar approach we get the formula.

Theorem 5 : For $\mathrm{g}=4, \mathrm{~N}\left(G_{5, m, 4}^{\prime}\right)=85 \times\left(\begin{array}{c}m \\ 4\end{array}\right), m \geq 4$ Proof :

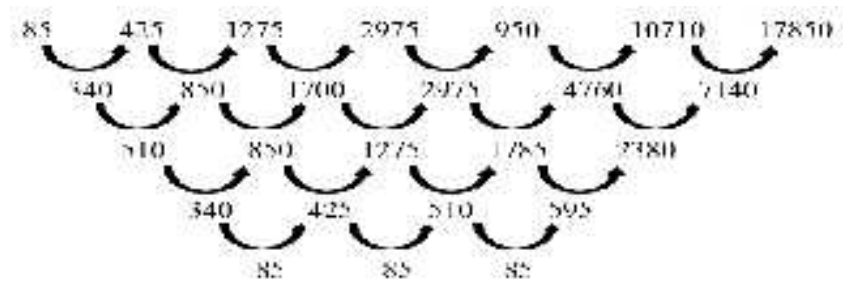

In this sequence we also notice the fixed difference in the sequence also occurs on the fourth order, so by similar approach we get the formula.

Theorem 6 : For $\mathrm{g}=5, \mathrm{~N}\left(G_{5, \mathrm{~m}, \mathrm{~L}}^{\prime}\right)=30 \times\left(\begin{array}{c}\mathrm{m-1} \\ 4\end{array}\right), m \geq 5$ Proof : 


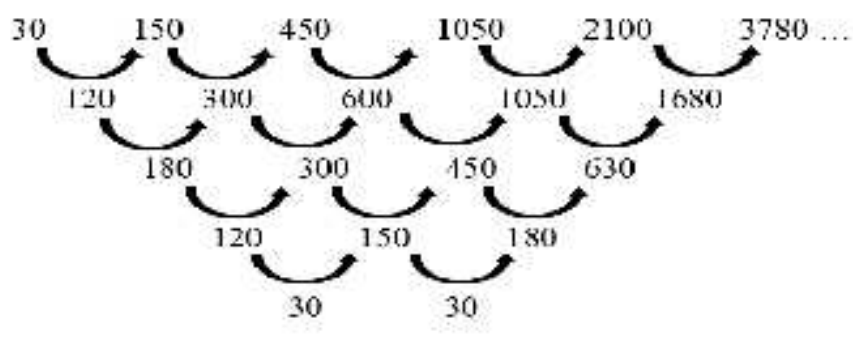

For $\mathrm{g}=5$, the fixed difference again appears on the fourth order, therefore by similar approach we get the formula.

Theorem 7 : For $g=6, N\left(G^{\prime}, m, \square\right)=5 \times\left(\begin{array}{c}m-2 \\ 4\end{array}\right), m \geq 6$

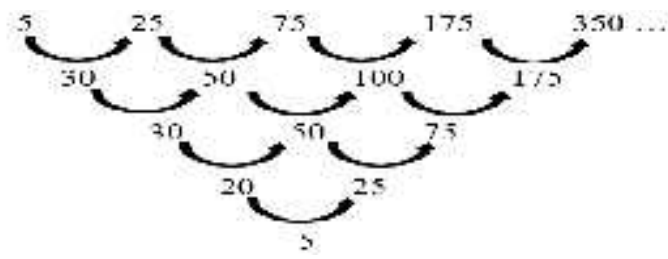

Finally, for $g=7$, the fixed difference in the sequence also appears on the fourth order, therefore the sequence is the arithmetics sequence of order four. Thus, by similar approach we get the formula.

Please be noted here that the fix number that occurs on the bottom of the sequence is the same number that appears as the multplication factor on the equation on Theorems 2 to 7 .

Therefore, the formula for counting the number of disconnected labeled graphs without parallel edges for $n=5$ and $m \geq 1$ is :

$$
\begin{aligned}
& \mathrm{N}\left(G^{r}{ }_{5, m, y}\right)=\mathrm{N}\left(G_{5, m, 0}^{r}\right)+\sum_{y-1}^{6} \mathrm{~N}\left(G^{\prime}{ }_{5, m, y}\right) \\
& =\mathrm{N}\left(G^{\prime}{ }_{5, l}\right)+\mathrm{N}\left(G^{\prime}{ }_{5, m, 1}\right)+\mathrm{N}\left(G^{\prime}{ }_{5, m, 2}\right) \\
& +\mathrm{N}\left(G_{5, \mathrm{~m}, 3}^{\prime}\right)+\mathrm{N}\left(G_{5, \mathrm{~m}, \mathrm{q}}^{\prime}\right)+\mathrm{N}\left(G_{5, \mathrm{~m}, 5}^{\prime}\right) \\
& +\mathrm{N}\left(G_{5, \mathrm{~m}, \mathrm{6}}^{\prime}\right) \\
& =\left(\begin{array}{c}
m+q \\
q
\end{array}\right)+10\left(\begin{array}{c}
m+3 \\
q
\end{array}\right)+45\left(\begin{array}{c}
m+2 \\
q
\end{array}\right)+120\left(\begin{array}{c}
m+1 \\
q
\end{array}\right) \\
& +85\left(\begin{array}{c}
m q \\
q
\end{array}\right)+30 \times\left(\begin{array}{c}
m-1 \\
q
\end{array}\right)+5\left(\begin{array}{c}
m-2 \\
q
\end{array}\right)
\end{aligned}
$$

\section{CONCLUSION}

From the above discussion we can conclude that the number of disconnected labeled graphs without paralel edges is

$$
\begin{aligned}
\mathrm{N}\left(G_{5, m, y}^{\prime}\right) & =\mathrm{N}\left(G_{5, m, 0}^{\prime}\right)+\sum_{y-1}^{6} \mathrm{~N}\left(G_{5, m, y}^{\prime}\right) \\
& =\left(\begin{array}{c}
m+q \\
q
\end{array}\right)+10\left(\begin{array}{c}
m+3 \\
q
\end{array}\right)+45\left(\begin{array}{c}
m+2 \\
q
\end{array}\right)+85\left(\begin{array}{c}
m \\
q
\end{array}\right) \\
& +30\left(\begin{array}{c}
m-1 \\
q
\end{array}\right)+5\left(\begin{array}{c}
m-2 \\
q
\end{array}\right)
\end{aligned}
$$

\section{REFERENCES}

[1] Foulds, L.R. Graph Theory Applications. Springer-Verlag, New York, USA, 1992

[2] Hsu, L.H., and Lin, C.K. Graph Theory and interconnection network. Taylor and Francis Group, LLC, New York, 2009.

[3] Cayley, A., 'On the Mathematical Theory of Isomers', Philosophical Magazine, vol. 47, no. 4, 1874, pp.444 - 446 ,

[4] Slomenski, W.F.(1964), 'Application of the Theory of Graph to Calculations of the Additive Structural Properties of Hydrocarbon', Russian Journal of Physical Chemistry, vol. 38, 1964, pp.700-703

[5] Harary F, and E. M. Palmer, Graphical Enumeration. Academic Press, New York, 1973

[6] Stanley, R.P ,. Enumerative Combinatorics, Vol. 1, number 49 of Cambridge Studies in Advanced Mathematics. Cambridge University Press, New York. 1997

[7] Stanley, R.P, Enumerative Combinatorics, Vol. 2, number 62 of Cambridge Studies in Advanced Mathematics. Cambridge University Press, New York, 1999.

[8[ Wilf, H, 1. Generating Functionology. Academic Press, second Edition, New York, 1994

[9] Agnarsson, G. and , R.D.Greenlaw, Graph Theory Modelling, Application, and Algorithms. Pearson/Prentice Education, Inc., New Jersey. 